\title{
Zum Geburtstag von Prof. Dr. Reinbert Schauer
}

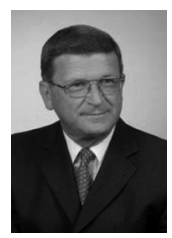

\begin{abstract}
Universitätsprofessor Dr. Reinbert Schauer von der Johannes Kepler Universität Linz feierte am 8. November 2013 seinen siebzigsten Geburtstag. Dies ist für die ZögU Anlass, nicht nur dem Jubilar ganz herzlich zu gratulieren, sondern seine ganz besonderen Lebensleistungen in Forschung, Lehre und Wissenstransfer auf den Gebieten des Rechnungswesens und Controllings öffentlicher Einrichtungen und privater Nonprofit-Organisationen zu würdigen.
\end{abstract}

Im Rahmen der umfassenden und breit gefächerten wissenschaftlichen Arbeiten und Aktivitäten von Reinbert Schauer stellen das Haushalts- und Rechnungswesen und das Controlling sowohl von öffentlichen Einrichtungen (speziell für öffentliche Verwaltungen) und von Nonprofit-Organisationen einen besondere Schwerpunkte dar. Dabei ist das Grundverständnis seiner Tätigkeit einerseits auf die praktische Anwendbarkeit theoretischer Erkenntnisse und andererseits auf einen theoretischen Bezug von Praxis gerichtet. Generell geht es ihm als Wissenschaftler in gesellschaftlicher Verantwortung um eine Theorie geleitete Praxis und eine Praxis geleitete Theorie, ein Verständnis, das sich auf sein gesamtes Lebenswerk erstreckt.

In diesem Sinne hat er gerade auf dem Gebiet des öffentlichen Haushalts- und Rechnungswesens die wissenschaftliche Entwicklung und die sich daraus ergebende praktische Reform im öffentlichen Sektor auf unterschiedlichen Ebenen national und international maßgeblich beeinflusst. So war es Reinbert Schauer, der als stellvertretender Vorsitzender (1985 bis 1987) und dann als Vorsitzender (1987 bis 1989) der Wissenschaftlichen Kommission „Öffentliche Unternehmen und Verwaltungen“ (Öffentliche Betriebswirtschaftslehre/Public Management) des Verbandes der Hochschullehrer für Betriebswirtschaft e. V. die Fachdiskussion um die wissenschaftlichen Grundlagen der Reform auf diesem Gebiet organisierte und ihr neue Impulse verlieh. Es gelang ihm, die Ansätze unterschiedlicher Reformkonzepte in Deutschland, in der Schweiz und in Österreich zusammenzuführen, Gemeinsamkeiten herauszuarbeiten und weiterzuentwickeln. Hieraus entstanden dann u. a. auch die unter Schauers Leitung im Namen der Kommission erarbeiteten "Leitlinien für die Reform des öffentlichen Rechnungswesens". ${ }^{1}$

Des Weiteren hat Schauer seine Forschungsarbeiten in Verbindung mit der Fachdiskussion in der wissenschaftlichen Kommission ganz konkret und konstruktiv genutzt, um die praktische Entwicklung in Österreich auf diesem Weg weiter voranzubringen. Er machte mit seinen Arbeiten deutlich, dass mit der Integrierten Haushaltsverrechnung auf (buchungstechnisch) doppischer Grundlage Österreich als erstes Land Erkenntnisse der betriebswirtschaftlichen Rechnungstheorie auf öffentliche Verwaltungen übertragen hat. In Österreich wurde vergleichsweise schon sehr früh

1 Nur am Rande sei erwähnt, dass unter Leitung von Reinbert Schauer sich die Kommission nicht nur mit dem Haushalts- und Rechnungswesen befasst hat, wenngleich dies doch sehr dominant war, sondern auch mit der für die Praxis und aufgrund der Rechtsprechung ganz wesentlichen Komplex des kommunalen Querverbundes. Auch hier war Schauer mit seinen Fachveröffentlichungen federführend. Beispielsweise sei auf den von ihm herausgegebenen Sammelband Schauer, Reinbert (Hrsg.): Der kommunale Querverbund aus der Sicht von Theorie und Praxis, Baden-Baden 1989 verwiesen). 
ein mehrdimensionales Rechnungswesen im Sinne der später in Deutschland für die Kommunen entwickelten 3-Komponenten-Rechnung praktiziert. Von daher ist es eines der Verdienste von Schauer, die bereits seit Ende der 1960er Jahre bestehende Pionierrolle Österreichs für eine wenn auch noch unvollständige - integrierte Verbundrechnung verdeutlicht zu haben, die z. B. in Deutschland erst in den 1980er Jahren in die Diskussion kam. Dabei setzte er sich vor allem auch mit den Gründen auseinander, weshalb die schon sehr früh entwickelten Elemente einer Finanzrechnung, einer Ergebnisrechnung und einer Vermögensrechnung und damit die in einigen zentralen Aspekten gegebene Vorreiterrolle Österreichs nur zum Teil und zu wenig für die praktische Steuerung von Verwaltungshandeln genutzt wurden. Hierauf aufbauend leistete er dann wesentliche Beiträge zur Weiterentwicklung des öffentlichen Haushalts- und Rechnungswesen, die weit über den Österreichischen Raum hinaus in die Fachdiskussion und praktische Entwicklung hineinwirkten.

In seinen Analysen kommt Schauer zu dem Schluss, das sich das öffentliche Haushalts- und Rechnungswesen vom traditionell an finanzwirtschaftlichen Größen und an der parlamentarischen Rechenschaftslegung ausgerichteten bisherigen Rechnungswesen zu einem umfassenderen Rechnungslegungs-, Steuerungs- und Führungsinstrument weiterentwickeln muss. Es geht ihm darum, das Kernelement der öffentlichen Steuerung, das Haushalts- und Rechnungswesen, jenen Anforderungen anzupassen, die durch das Konzept des (New) Public Management bzw. der Wirkungsorientierten Verwaltungsführung (WOV) vorgegeben sind. Diese lenken den zentralen Fokus neben einer ressourcenorientierten Steuerung auf die mit den bereitgestellten Ressourcen zu erstellenden Leistungen bzw. angestrebten Wirkungen. Damit ist nicht nur die Forderung nach mehr Effektivität und (Produktions- sowie allokative) Effizienz im Verwaltungshandeln und nach mehr Transparenz in Bezug auf die gesetzten politischen Ziele, den Ressourcenverbrauch, die finanzielle Lage von Gebietskörperschaften und im Hinblick auf die Verwendung öffentlicher Mittel verbunden, sondern auch die Forderung nach einer operationalen Handhabung der intergenerativen Gerechtigkeit. Dieses gesellschaftspolitische Problem bezieht sich auf die Tendenz der derzeitigen Generation, die finanziellen Lasten aktueller politischer und gesellschaftlicher Ziele mithilfe der Verschuldung auf kommende Generationen zu verlagern - ein Aspekt, der nicht nur in der wissenschaftlichen, sondern auch schon in der alltäglichen Diskussion einen immer breiteren Raum einnimmt. Hier wird beispielhaft deutlich, wie stark Schauers Forschungsarbeiten nicht im Abstrakten und Theoretischen verhaftet bleiben, sondern sich darüber hinausgehend unmittelbar und ganz konkret mit hoch aktuellen gesellschaftspolitischen Problemen befassen.

Insgesamt hat Reinbert Schauer die Bemühungen um die Reform- und Neugestaltung des öffentlichen Rechnungswesens - sowohl im Hinblick auf die nationalen als auch die transnationalen Entwicklungen - in ganz entscheidenden Maße mitgeprägt. Davon zeugen nicht nur die Vielzahl seiner einschlägigen Veröffentlichungen und wissenschaftlichen Initiativen, sondern nicht zuletzt auch seine Tätigkeit als wissenschaftlicher Berater der politischen und administrativen Entscheidungsträger im Rahmen einzelner konkreter Reforminitiativen. Er hat insbesondere auch österreichische Spezifika und Vorreiterrollen in der Reformentwicklung und damit auch die „österreichische Sicht der Dinge“ in die internationale Diskussion. Damit hat er für einen zentralen Problemkomplex des öffentlichen Sektors - trotz dessen starker Bindung an nationalstaatliche (gesetzliche) Rahmenbedingungen - wesentliche Impulse für den gesamten mitteleuropäischen Raum gesetzt. Auf Österreich bezogen, ist er als ein zentraler wissenschaftlicher Motor, Initiator, 
aber auch als kritischer Mahner der Bemühungen um ein neues öffentliches Haushalts- und Rechnungswesen anzusehen, der den Diskussionsprozess bis zum heutigen Tage in Gang hält und die praktische Umsetzung der Reformideen wissenschaftlich begleitet. In diesem Sinne ist auch die aktuelle und tiefgreifende Reform auf Bundesebene in Österreich nicht unwesentlich vom Wirken und von den Aktivitäten Schauers beeinflusst.

Die wissenschaftlichen Tätigkeiten von Reinbert Schauer haben jedoch nicht nur dem Rechnungswesen und Controlling öffentlicher Einrichtungen wesentliche Impulse geliefert, sondern auch dem privater Nonprofit-Organisationen. So hat er die fortschreitende Umstrukturierung eines traditionell nur an finanzwirtschaftlichen Größen ausgerichteten Rechnungswesens zu einem innovativen Instrument eines modernen und erfolgsorientierten Verbands- bzw. NPO-Managements konzeptionell entscheidend mitgeprägt. Analog zum öffentlichen Sektor war auch im Bereich der privaten Nonprofit-Organisationen das System der Ermittlungsrechnungen in Richtung eines 3-Komponenten-Rechnungswesens zu erweitern und um spezifische Instrumente des entscheidungs- und steuerungsorientierten Rechnungswesens zu ergänzen. Da aufgrund der Missions- und der damit verbundenen Sachzielorientierung auch in Nonprofit-Organisationen Erfolg nicht adäquat an finanziellen Größen gemessen werden kann, sind diese durch nicht finanzielle Indikatoren und Kennzahlen zu ergänzen, die ihrerseits wiederum einen zielorientierten Aufbau von statistischen Daten in eigenen Mengenrechnungen voraussetzen. Die für den NPO-Bereich entwickelten und über die Jahre hinweg verfeinerten Instrumente bauen zwar vielfach auf ähnlichen Grundmustern wie im Verwaltungsbereich auf, sind aber dennoch an die spezifischen Besonderheiten privater Nonprofit-Organisation anzupassen. In diesem Zusammenhang ist insbesondere auch darauf hinzuweisen, dass die bedeutsamen und richtungsweisenden Ergebnisse der Forschung von Reinbert Schauer in vielfacher Weise in das Freiburger Management-Modell für Nonprofit-Organisationen Eingang gefunden haben und dessen ständige Weiterentwicklung in den Bereichen Rechnungswesen, Controlling und Finanzierung bzw. Ressourcenmanagement wesentlich mitgestalten.

Wie sehr Schauer die Diskussion in der Scientific Community prägt, schlägt sich in zahlreichen Kooperationen und Forschungskolloquien mit anderen Universitäten und den hieraus hervorgegangenen Sammel- und Tagungsbänden nieder. Verwiesen sie hier etwa auf die Internationalen Colloquien der NPO-Forscher und die über weit mehr als ein Jahrzehnt regelmäßig durchgeführten Forschungskolloquien mit den Universitäten Freiburg (Schweiz), Bern, Hamburg und Potsdam. Als Mittler zwischen Wissenschaft und Praxis organisiert Reinbert Schauer Fachtagungen und Weiterbildungsveranstaltungen, die Praktikern aus dem Bereich der öffentlichen Verwaltung und Nonprofit-Organisationen den unmittelbaren Zugang zu aktuellem universitären Wissen ermöglichen.

Reinbert Schauer ist einer der ganz wenigen Fachvertreter, die durch Ihr wissenschaftliches Wirken die Entwicklung der Fachgebiete Public und Nonprofit Management ganz maßgebend beeinflusst haben und mit dem Erkenntnistransfer in die Praxis für die neueren Reformen in Staat, Verwaltungen und im Nonprofit Sektor für den deutschsprachigen Bereich prägend waren. Nicht zuletzt ist Schauers vorbildliches - heute keineswegs mehr alltägliches - Berufsethos als Hochschullehrer zu erwähnen, das Studierende, Mitarbeiter und Kollegen gleichermaßen immer wieder beeindruckt.

Wir wünschen uns und ihm, dass er noch viele Jahre sein bisheriges Wirken fortsetzen kann. 